\title{
The effects of self-perception and perceptual contrast upon compliance with socially undesirable requests
}

\author{
MITRI E. SHANAB and PAMELA J. O'NEILL \\ California State University, Fresno, California 93740
}

\begin{abstract}
The relative effects of self-perception and perceptual contrast upon rate of compliance with a counternormative request were studied in a 5 by 2 by 2 design that combined five sizes of the initial request, two levels of authority, and sex. Increased compliance was obtained only when the initial requests were either moderately small or excessively large, but not when they were either very small or moderately large.
\end{abstract}

Without the aid of force, individuals can, upon occasion, be influenced to comply with counternormative requests. The present study sought to investigate the rate of compliance with socially undesirable requests, with three objectives in mind: (1) to compare the two methods (foot in the door, FID, and door in the face, DIF) that have been used to facilitate compliance, (2) to check the adequacy of predictions based upon the two theories of self-perception and perceptual contrast, and (3) to investigate the role of authority in compliance.

According to the FID technique, subjects are first presented a small request that has a high probability of acceptance and are then confronted with a larger request. Conversely, the DIF strategy starts with an unreasonably large request that normally elicits refusal and is then followed by a more moderate request. These sequential request strategies have been shown to be effective in increasing the frequency of compliance with the second, critical request.

The compliance observed in the FID technique has been explained in terms of Bem's (1972) self-perception theory. According to this theory, people infer their feelings and attitudes through observation of their behavior. Thus, subjects who comply with an initial request would presumably explain such behavior by attributing it to an underlying compliant disposition, especially since no apparent external forces are present. When confronted with a second, larger request, these subjects would, therefore, be expected to show a higher rate of compliance than control subjects faced with only the second request.

The self-perception theory leads to the prediction that not only does compliance lead to further compliance, but noncompliance should also lead to subsequent noncompliance. This prediction is partially borne out

Portions of this paper, which is based on the second author's master's thesis, were read by the first author at the 22 nd annual meeting of the Psychonomic Society, held in Philadelphia, November 12-14, 1981. by the results of two DIF studies in which noncompliance with the initial large request led to noncompliance with a moderate request presented after a delay of either 2 days (Snyder \& Cunningham, 1975) or 7-10 days (Cann, Sherman, \& Elkes, 1975). When the second request follows the first one without any delay, the results have repeatedly shown an increase in compliance. Such a finding is obviously inconsistent with selfperception theory. Cialdini, Vincent, Lewis, Catalan, Wheeler, and Darby (1975) argued that the increase in compliance with the second request is best explained in terms of a reciprocal concessions model. If, following rejection of an initial demanding request, the experimenter immediately presents an alternative request that is considerably less demanding, the latter request might be construed as representing a concession on the part of the experimenter, and the subject would probably reciprocate by conceding to the critical request.

Miller, Seligman, Clark, and Bush (1976) argued that in studies based on the rejection-then-moderation technique, which is involved in the DIF paradigm, the effects of reciprocal concessions and perceptual contrast are usually confounded. When a large request is subsequently reduced to a more reasonable request, the subject might perceive this action as a concession made by the requester and, therefore, feel obligated to reciprocate. On the other hand, compliance might be increased because of contrast effects resulting from a perceived gain (or decreased loss) for the target subject. Miller et al. (1976) separated the effects of these factors and examined the role played by each in mediating compliance. They found that the critical factor inducing compliance was perceived contrast. Apparently, individuals weigh the potential gain, or decreased loss, derived from agreeing to a certain critical request against some standard or anchor established by a preceding request. When compared against such an anchor, the critical request seems much smaller than it would otherwise appear, thus facilitating compliance.

Using socially undesirable requests in a replication of 
Miller et al.'s (1976) design, Shanab and O'Neill (1979) also found that the critical determinant of compliance was the perceived contrast between the initial unreasonable or high-cost request and the subsequent less demanding or low-cost request. Shanab and Isonio (1980) replicated the main features of the Shanab and O'Neill study, except that the critical request was presented 5-14 days following the first request. No significant increase in compliance was observed. Taken together, the two studies seem to imply that contrast is probably both a necessary and a sufficient condition for increased compliance in the DIF paradigm.

The perceived authority of the experimenter is most often cited as the critical factor eliciting compliance in obedience research that also employs counternormative requests. Compliance could be considered a special case of obedience, since the requester represents some form of authority (cf. Shanab \& O'Neill, 1979). This might imply that authority is a sufficient condition for compliance, but whether or not it is a necessary condition for compliance cannot be determined with certainty since there are no known studies that manipulated degree of authority in either paradigm.

Another factor that appears to be crucial in facilitating compliance is the size of the initial request. Seligman, Bush, and Kirsch (1976) reported that compliance in the FID paradigm was an increasing function of the size of the initial request. No compliance was observed when the initial request was too small because it was felt that the very small size of the request made it difficult for the subjects to form appropriate self-perceptions. However, another process, namely contrast, could be operating both when the initial requests are very small and when they are excessively large. When the first request is very small and the subject consents, the second request would, by comparison, look much larger than it actually is and the subject would refuse to carry out the favor. Similarly, a second large request, presented immediately following an unreasonably large request, would be perceived as smaller or less demanding than it actually is and would, the refore, lead to acceptance.

When the initial request is either not exceedingly small or large, however, the self-perception process could be involved. If the first request is small enough to lead to acceptance, the subject would be likely to attribute this acceptance to an underlying compliant disposition and, thus, would be more likely to comply with the second, larger request. If the initial request is large enough (but not excessively so) for the subject to reject it, then a more moderate second request would also be rejected, because rejection of the first request would be attributed by the subject to an underlying noncompliant disposition.

In summary, it was hypothesized that a significant increase in compliance would be obtained in the FID paradigm when the initial request is moderately small but not when it is exceedingly small. Similarly, an increase in compliance would be expected in the DIF paradigm when an extremely large but not simply large request precedes the more moderate critical request. Moreover, the increase in compliance in both paradigms would be more pronounced when the requester appears as a high- as opposed to a low-authority figure. No specific predictions were made for any differential effects due to sex.

\section{METHOD}

\section{Design}

A 5 by 2 by 2 design that combined five sizes of the initial request (very small, small, large, very large, and none), two levels of authority (high vs. low), and sex was used. All subjects were presented the same critical request.

\section{Subjects}

The subjects were 200 undergraduate students enrolled in an introductory psychology class at California State University, Fresno. The subjects were randomly assigned to each subgroup.

\section{Procedure}

The same female experimenter played both roles of authority. All experimental subjects were presented one of four differentsize initial requests. All subjects were told that they would be involved in an experiment that required the administration of punishment in the form of a mild electric shock to either human or animal subjects participating in a learning task. Control subjects were not presented the initial request.

In the "very large" condition, the subjects were asked if they would volunteer to be in a study that would require them to spend $2 \mathrm{~h} /$ day for 2 months, during which their main task would be to administer punishment to human subjects. In the "large" condition, the subjects received essentially the same request, except that they were to spend $1 \mathrm{~h} /$ day for 1 week. The last two conditions constitute the DIF manipulation. In the "small" condition, the subjects were asked if they would spend only $15 \mathrm{~min}$, during which they would be required to punish rats. In the "very small" condition, the subjects were asked if they would spend a minute or so carrying a couple of rats in their cages to a nearby room in which an experiment was being conducted investigating the effects upon learning of punishing rats with mild electric shocks. The last two conditions reflect the FID manipulation. The experimental subjects received the critical request immediately following their responses to the first request. All subjects, including the controls, received the same critical request, which consisted of asking them to volunteer for another study that required only $1 \mathrm{~h}$ of their time and involved punishing dogs with a mild electric shock. At the conclusion of the experiment, the subjects were debriefed en masse.

\section{RESULTS AND DISCUSSION}

The data were first analyzed by means of log-linear models. Neither the sex factor nor the authority factor yielded significant results, but the size of the initial request did. The rates of compliance in the FID manipulation were $25 \%$ for the very small initial request condition and $70 \%$ for the small request condition. In the DIF conditions, $35 \%$ of the subjects complied when the initial request was moderately large, but $72.5 \%$ complied when it was excessively large. When compared with the $30 \%$ compliance rate displayed by the controls, a significant FID effect was observed only when a small initial request was used $\left[\chi^{2}(1)=11.25, p<.001\right]$, but not when it was very small $\left[\chi^{2}(1)<1\right]$. Similarly, a significant DIF phenomenon was obtained when a very large initial request was used $\left[\chi^{2}(1)=12.81, \mathrm{p}<.001\right]$, but not 
when it was merely large $\left[\chi^{2}(1)<1\right]$. Correction for continuity was carried out in all chi-square analyses.

The results clearly show that the increase in compliance was a function of the relative size of the initial request and not necessarily the particular technique used. Holding the critical request constant in both paradigms, a very small initial request does not seem to lead to increased compliance but a moderately small request does, and a very large (and unreasonable) initial request does lead to increased compliance but a moderately large one does not. It was posited earlier that contrast determined the occurrence or nonoccurrence of compliance when the initial request was either very small or very large. Apparently, the subject in such situations focuses attention on the size of the request and either accepts it when it is very small or rejects it when it is very large. In the first case, the critical request would look much larger, or be judged more demanding, than it actually is and would, therefore, lead the subject to reject it. In the second case, in which the initial request is very large, the critical request would look much smaller, or be judged less demanding, than it actually is and would thus lead the subject to accept it.

Self-perception, on the other hand, was assumed to be involved when the initial request was either moderately small or moderately large. This is because the formation of appropriate self-perceptions would be facilitated in such situations, in which the initial request is neither trivial nor unreasonable. When the first request is moderately small, the critical request would be accepted because subjects would attribute their acceptance of it to an underlying compliant disposition. Compliance with the second request would thus be consistent with such selfperception. When the initial request is large enough (but not excessively so) that subjects would reject it, they would attribute such refusal to an underlying noncompliant disposition and, thus, would be more likely not to comply with the critical request. In the interest of parsimony, the last two cases could be explained in terms of contrast rather than self-perception. In both cases, one can argue, contrast is diminished, and thus there would be no reason for subjects to respond differently to the critical request. In the FID condition, after agreeing to comply with a moderately small request, the subjects would be more likely to consent to the critical request because the two requests would be perceived as similar in size, and hence, agreeing to comply with the critical request would not be perceived as involving any loss. Similarly, rejection of the moderately large initial request in the DIF condition would lead to a similar rejection of critical request, not because the subjects make a dispositional inference about themselves, but because they perceive the two requests as being similar in size, which, in turn, could lead them to perceive no particular gain in accepting the critical request.

The present results indicate that perceived authority apparently does not influence compliance as it does obedience. Unlike the obedience paradigm, the subject in the compliance paradigm is not under any form of pressure to comply. It seems likely that different processes are involved in the two situations.

In summary, it appears that compliance with a critical second request is, in large part, determined by the perceived size of the initial request.

\section{REFERENCES}

BEM, D. J. Self-perception theory. In L. Berkowitz (Ed.), Advances in experimental social psychology (Vol. 6). New York: Academic Press, 1972.

Cann, A., Sherman, S. J., \& Elkes, R. Effects of initial request size and timing of a second request on compliance: The foot-in-the-door and the door-in-the-face. Journal of Personality and Social Psychology, 1975, 32, 774-782.

Cialdini, R. S., Vincent, J. E., Lewis, J. K., Catalan, J., Wheeler, D., \& DARBY, B. L. Reciprocal concessions procedure for inducing compliance: The door-in-the-face technique. Journal of Personality and Social Psychology, 1975, 31, 206-215.

Miller, R. L., Seligman, C., Clark, N. T., \& Bush, M. Perceptual contrast versus reciprocal concessions as mediators of induced compliance. Canadian Journal of Behavioural Science, 1976, 33, 517-520.

Seligman, C., Bush, M., \& Kirsch, K. Relationship between compliance and the foot-in-the-door paradigm and the size of the first request. Journal of Personality and Social Psychology, 1976, 33, 517-520.

Shanab, M. E., \& Isonio, S. A. The effects of delay upon compliance with socially undesirable requests in the door-inthe-face paradigm. Bulletin of the Psychonomic Society, 1980, 15, 76-78.

Shanab, M. E., \& O'Neill, P. J. The effects of contrast upon compliance with socially undesirable requests in the door-in-theface paradigm. Canadian Journal of Behavioural Science, 1979, 11, 236-244.

Snyder, M., \& Cunningham, M. R. To comply or not to comply: Testing the self-perception explanation of the "foot-in-thedoor" phenomenon. Journal of Personality and Social Psychology, 1975, 31, 64-67.

(Received for publication February 17, 1982.) 